University of Nebraska - Lincoln

DigitalCommons@University of Nebraska - Lincoln

Faculty Publications from the Department of Electrical \& Computer Engineering, Department Electrical and Computer Engineering

$3-1-2000$

\title{
In situ ellipsometry growth characterization of dual ion beam deposited boron nitride thin films
}

\author{
E. Franke \\ University of Nebraska-Lincoln, efranke3@unl.edu \\ Mathias Schubert \\ University of Nebraska-Lincoln, mschubert4@unl.edu \\ John A. Woollam \\ University of Nebraska-Lincoln, jwoollam1@unl.edu \\ J.-D. Hecht \\ Institut fu"r Oberfla“chenmodifizierung Leipzig e.V., Permoserstrasse 15, 04318 Leipzig, Germany \\ G. Wagner \\ Institut fu"r Oberfla“chenmodifizierung Leipzig e.V., Permoserstrasse 15, 04318 Leipzig, Germany \\ See next page for additional authors
}

Follow this and additional works at: https://digitalcommons.unl.edu/electricalengineeringfacpub

Part of the Electrical and Computer Engineering Commons

Franke, E.; Schubert, Mathias; Woollam, John A.; Hecht, J.-D.; Wagner, G.; Neumann, H.; and Bigl, F., "In situ ellipsometry growth characterization of dual ion beam deposited boron nitride thin films" (2000). Faculty Publications from the Department of Electrical and Computer Engineering. 19.

https://digitalcommons.unl.edu/electricalengineeringfacpub/19

This Article is brought to you for free and open access by the Electrical \& Computer Engineering, Department of at DigitalCommons@University of Nebraska - Lincoln. It has been accepted for inclusion in Faculty Publications from the Department of Electrical and Computer Engineering by an authorized administrator of DigitalCommons@University of Nebraska - Lincoln. 
Authors

E. Franke, Mathias Schubert, John A. Woollam, J.-D. Hecht, G. Wagner, H. Neumann, and F. Bigl 


\title{
In situ ellipsometry growth characterization of dual ion beam deposited boron nitride thin films
}

\author{
E. Franke, $\left.{ }^{a}\right)$ M. Schubert, and J. A. Woollam \\ Center for Microelectronic and Optical Materials Research, and Department of Electrical Engineering, \\ University of Nebraska, Lincoln, Nebraska 68588-051 \\ J.-D. Hecht, G. Wagner, H. Neumann, and F. Bigl \\ Institut für Oberflächenmodifizierung Leipzig e.V., Permoserstrasse 15, 04318 Leipzig, Germany
}

(Received 21 September 1999; accepted for publication 3 December 1999)

\begin{abstract}
Pure hexagonal $h$, as well as mixed-phase cubic/hexagonal $c / h$ boron nitride (BN) thin films were deposited onto [001] silicon substrates using the dual ion beam deposition technique. The BN thin films were grown under UHV conditions at different substrate temperatures and ion beam bombarding parameters. Thin-film growth was monitored using in situ spectroscopic ellipsometry at 44 wavelengths between 420 and $761 \mathrm{~nm}$. The in situ ellipsometric $\Psi$ and $\Delta$ data were compared with two-layer growth model calculations for the mixed-phase $c / h \mathrm{BN}$, and with one-layer growth model calculations for pure $h$-BN growth. In situ data provide information on the optical properties of deposited $h$ - $\mathrm{BN}$ and $c / h$ - $\mathrm{BN}$ material, film thickness, and $\mathrm{BN}$ growth rates. A virtual interface approach is employed for the optical properties of the silicon substrate. The growth and nucleation of $c$-BN observed here confirms the cylindrical thermal spike model. The results for composition and thickness of the BN films were compared to those obtained from ex situ infrared transmission measurements and high-resolution transmission electron microscopy investigations. (c) 2000
\end{abstract} American Institute of Physics. [S0021-8979(00)08705-3]

\section{INTRODUCTION}

In the past years boron nitride (BN) has gained an increasing interest as thin-film material due to its extreme mechanical hardness. Boron nitride is known as a polymorphic compound. Four modifications: a rhombohedrical, two hexagonal, and a cubic have been observed. However, in thinfilm deposition only two BN modifications, a hexagonal $h$ with graphite-like layer structure, and a cubic $c$ modification with zincblende structure is commonly observed. The $s p^{2}$-bonded $h$-BN is a relatively soft, electrically insulating material, which is birefringent and transparent in the visible spectral region. The wide band gap $s p^{3}$-bonded $c$-BN material is electrically insulating, transparent in the visible spectral region, and the second hardest material (only diamond is harder). The ability to manufacture $c$-BN thin films would open up wide ranging applications, such as for optical or abrasive coatings. ${ }^{1}$

Recently, various ion-assisted chemical, ${ }^{2,3}$ and physical vapor phase ${ }^{4-7}$ deposition techniques were used to grow polycrystalline $\mathrm{BN}$ thin films. Regardless of the growth method, these films are similar in microstructure due to a characteristic layer growth. ${ }^{8}$ In the first step of the BN thinfilm growth a few $\mathrm{nm}$ thick amorphous (a) BN layer is created, caused by ion mixing on top of the silicon $(\mathrm{Si})$ substrate. This amorphous layer consists of the elements boron, nitrogen, $\mathrm{Si}$, and oxygen. ${ }^{6}$ The $a$-BN layer is covered during the continuing deposition process by a highly textured $h$-BN nucleation layer. Depending on the strength of the ion bom-

\footnotetext{
a) Author to whom correspondence should be addressed; electronic mail: franke@engrs.unl.edu
}

bardment, the $h$ - $\mathrm{BN}$ growth continues or the nucleation layer can be overgrown by a nanocrystalline $c$-BN layer. This $c$-BN layer still contains $s p^{2}$-bonded material between the $c$-BN grains. ${ }^{9}$ Some authors have reported on a $\sim 0.2-\sim 0.4$ $\mathrm{nm}$ thick $s p^{2}$-bonded surface layer that terminates the polycrystalline $c$-BN film. ${ }^{4,10}$

Deposition of $c$-BN is limited by resputtering effects caused at high-energetic ion bombardment of the growing film, and by the low-energetic ion bombardment which causes $h$-BN growth only. Growth of $c$ - BN occurs for the intermediate strength of the bombarding ions, i.e., below the resputter region, and above the $h$ - $\mathrm{BN}$ regime.

Many theoretical attempts have been undertaken recently to understand the above mentioned peculiarities of the $\mathrm{BN}$ thin-film growth mechanism, and several models were suggested. ${ }^{11-17}$ The stress model explains $c$-BN film growth due to the high compressive stress in thin films. ${ }^{11-14}$ Another model explains $c$-BN formation caused by a preferential sputtering of the $s p^{2}$-bonded material during deposition. ${ }^{15}$ Thermal-spike theory suggests local recrystallization processes, which cause formation of nanocrystalline $c$-BN grains. ${ }^{16,17}$ None of the growth models developed so far can explain the microstructure in $\mathrm{BN}$ thin films and their dependence on the deposition parameters, although the models shed some light on BN formation during film growth.

Ellipsometry is known as a precise and nondestructive optical tool that allows for measurement of the complex ratio $\rho$ of the reflection coefficients $R_{p}$ and $R_{s}$ for incident light polarized parallel $p$ and perpendicular $s$ to the plane of incidence, respectively. ${ }^{18}$ The ellipsometric parameters $\Psi$ and $\Delta$ are defined as 


$$
\rho=R_{p} / R_{s}=\tan \Psi \exp \{i \Delta\},
$$

and contain spectral information on the optical and structural properties of thin-film samples. In general, a mathematical model is necessary for experimental data analysis. This model should include the physical material properties such as the refractive indices, the absorption coefficients, and if applicable, the optical birefringence. The model also includes the sample geometry, i.e., film thickness, multilayer structure, and direction of possible anisotropy. Ex situ ellipsometry was recently used to determine the isotropic $c$ - BN (e.g., at $\left.\lambda=571 \mathrm{nm:} n_{c}=2.1\right)$, and anisotropic $h$-BN $\left(n_{e}\right.$ $\left.=1.65, n_{o}=2.1\right)$ optical constants and thin-film layer structure of magnetron sputtered $\mathrm{BN} .{ }^{19-21}$. It was observed that the $h$-BN and $c$-BN thin-film optical constants are very similar to their bulk values. ${ }^{22}$

In situ spectroscopic ellipsometry allows real-time monitoring of thin-film growth. This technique is very sensitive to changes of the optical properties, or the growth rates of a growing thin film. In addition to information about optical and structural film properties, analysis of time-dependent in situ data provides nuclei formation, film coalescence, and growth rates, and allows on-line control of multiple-layer growth. ${ }^{23}$

Infrared (IR) spectroscopy is widely used to estimate the phase content of BN films. Transmission or reflectance spectra contain resonance peaks at the phonon modes of the BN modifications, and provide information on phase content within the thin films. Because of the uniaxial crystal structure, the IR spectra of polycrystalline $h$-BN films reveal two phonon bands at $\omega_{\mathrm{TO}(h)}=775 \mathrm{~cm}^{-1}$, and $\omega_{\mathrm{TO}(h)}$ $=1395 \mathrm{~cm}^{-1},{ }^{24}$ whereas the isotropic $c$-BN phonon mode is located at $\omega_{\mathrm{TO}(c)}=1080 \mathrm{~cm}^{-1} .25$

In this article we focus on the in situ spectroscopic ellipsometry study of ion-beam deposited BN thin films. The BN thin-film growth onto [001] Si substrates was performed at growth temperatures between 100 and $800^{\circ} \mathrm{C}$. In particular, we study the temperature dependence of $h$-BN vs $c$-BN thin-film growth. We also focus on the temperature dependence of the $h$ - $\mathrm{BN}$ and $c$ - $\mathrm{BN}$ growth rates. We obtain composition and thickness of the $\mathrm{BN}$ samples. We compare the compositions and thicknesses to those obtained from IR transmission measurements and high-resolution transmission electron microscopy (HR-TEM) images, respectively. The results obtained are discussed within the cylindrical thermal spike model.

\section{EXPERIMENT}

The BN thin films were deposited under ultrahigh vacuum conditions (typical background pressure $p=1$ $\times 10^{-9}$ Torr) using the dual ion beam technique. The deposition chamber is depicted in Fig. 1. Two $40 \mathrm{~mm}$ double-grid broad-beam Kaufman ion sources were attached to the deposition chamber in order to sputter from a pyrolytic $h$-BN target (ion beam sputtering), and to bombard the growing BN film (ion beam assisted). The sputtering and the irradiating ion sources were operated at a process pressure of $p$ $=7 \times 10^{-4}$ Torr using pure argon, and an argon/nitrogen mixture 1:1, respectively. The ion beam energy was adjust-

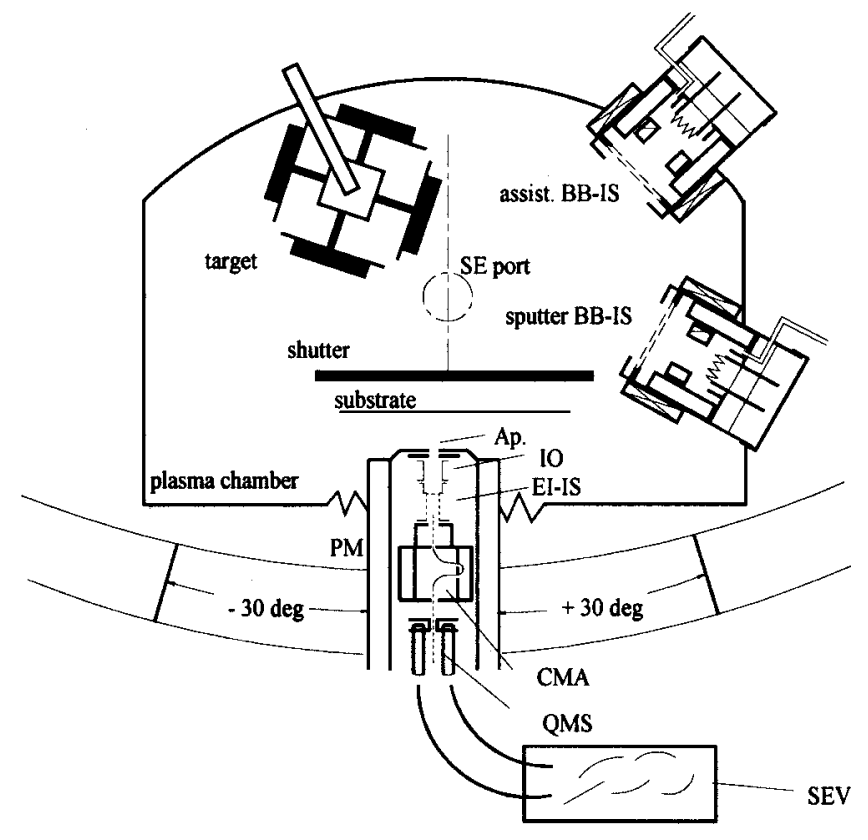

FIG. 1. UHV setup for the dual ion beam thin-film deposition of BN used in this work (not to scale). Two broad-beam Kaufman ion sources (BB-IS) are attached to the plasma chamber. The integrated plasma monitor consists of an aperture (Ap), ion optics (IO), electron impact ion source (EI-IS), modified cylindrical mirror analyzer (CMA), quadrupol mass spectrometer (QMS), secondary electron multiplier (SEV), and can be rotated by $\pm 30^{\circ}$. The plane of incidence for the in situ ellipsometer setup is perpendicular to this depicted plane and is indicated by the dashed-dotted line. Note that the deposition geometry leads to a linear nonuniform film thickness $\delta d / d$.

able between 50 and $1200 \mathrm{eV}$. The maximum ion beam current density was measured as $1.5 \mu \mathrm{A} / \mathrm{cm}^{2}$. Both ion sputtering, and irradiating beams were previously characterized by energy-resolved mass spectrometry (plasma monitoring), ${ }^{26}$ and by Faraday-cup measurements. The composition of both single and multiple charged ions within the ion beams, their kinetic energy distribution, and ion-current distributions were characterized as a function of ion source parameters. ${ }^{27}$ The sputtered neutral B and N particles were also characterized by plasma monitoring, and quartz microbalance measurements. Thereby we obtained the deposition rates, and the energy and mass of sputtered neutral particles. ${ }^{26}$

A 44-wavelength ellipsometer $(\lambda=420 \ldots 761 \mathrm{~nm})$ was attached to the deposition chamber at a fixed angle of incidence $\left(\varphi=74.3^{\circ}\right)$ for in situ monitoring of the BN thin film growth. The angle of incidence was calculated from the in situ spectroscopic ellipsometry scan taken on a silicon substrate before each deposition, using a standard numerical regression method. ${ }^{28}$ Here, only the thickness of the native silicon oxide layer and the angle of incidence were varied. The silicon and silicon dioxide optical functions were taken from tabulated reference data. ${ }^{29}$ During deposition, the substrate was heated by an electrical resistance heater. The temperature was adjustable between room temperature and $1200^{\circ} \mathrm{C}$, and was determined by a type-K (alumel-chromel) thermocouple attached to the resistance heater. Prior to $\mathrm{BN}$ deposition, the temperature dependence of the thermocouple output was calibrated by the in situ ellipsometric setup. A standard GaAs substrate was used to measure the near surface temperature of the wafer. Here, the temperature- 
dependent shift of the GaAs optical response was monitored, and compared to reference data of the temperature dependence of the GaAs dielectric function. ${ }^{30}$

In situ ellipsometric measurements were taken every 20 $\mathrm{s}$ during film growth. This time interval was found to be the optimum choice to account for both the accuracy and realtime thin-film growth monitor measurement. However, in this measurement mode we are not able to study fast nucleation processes or thin film coalescence.

One-side polished [001] silicon wafers were used as substrate material. Further ex situ characterization of BN samples was performed by HR-TEM, diffraction-contrast TEM, and selected-area electron diffraction at [110]Si/BN thin-film cross sections using a $200 \mathrm{keV}$ Phillips electron microscope. Fourier-transform IR (FTIR) transmission spectroscopy was used to obtain additional information on phase content of the BN films.

\section{RESULTS AND DISCUSSION}

Figures 2(a) and 2(b) show the time dependence of the in situ spectroscopic $\Psi$ and $\Delta$ spectra for two BN films [Fig. 2(a): $h$-BN; Fig. 2(b): $h / c-\mathrm{BN}]$. In order to avoid confusion, experimental data (symbols), and best-fit calculations (solid lines) of selected wavelengths are included for presentation only $(420,506,554,626,674,705,761 \mathrm{~nm})$. Figure 2(a) presents the ellipsometic spectra measured during growth of sample BN51. The temperature during growth was $T_{g}$ $=100{ }^{\circ} \mathrm{C}$ (Table I). At $t=0[A$ in Fig. 2(a)], the initialization of the deposition process caused rapid changes of the $\Psi$ and $\Delta$ spectra. After about 2.5 min $[B$ in Fig. 2(a)], film deposition began with a constant growth rate, producing a smooth evolution of the $\Psi$ and $\Delta$ spectra versus time. After about 45 min both ion sputter, and assist sources were interrupted to stop the growth process $[C$ in Fig. 2(a)]. Between $A$ and $C$, the process parameters were kept constant and a $81 \mathrm{~nm}$ thick $h$-BN film was deposited between $B$ and $C$. Within $A$ and $B$, the ion bombardment modified the surface of the silicon/ silicon dioxide substrate. As will be shown later, an amorphous layer with few $\mathrm{nm}$ thickness was created during this deposition period. Attempts to model the optical response of the sample between $A$ and $B$ failed. This is explained by the fast and complex changes which the surface undergoes at the beginning of the deposition process. However, consideration of the optical properties of the substrate is necessary for analysis of film properties. A method to overcome this problem is the virtual interface (VI) approach. ${ }^{31}$ In this approximation, the optical response of the sample at an appropriate time is converted into that of a virtual substrate material, assuming an ideal surface with no overlayer. Therefore, we did not explicitly consider the formation of the $a$-BN layer in the initial stage of film growth, and ellipsometric spectra at $t \sim 2.5$ min were used to calculate the optical properties of a VI.

Figure 2(b) presents $\Psi$ and $\Delta$ spectra measured during mixed-phase $h / c$-BN film growth of sample BN54 at $T_{g}$ $=400{ }^{\circ} \mathrm{C}$ (Table I). Initialization of the deposition process caused similar changes in $\Psi$ and $\Delta$ spectra as in Fig. 2(a) [A in Fig. 2(b)]. At $B^{\prime}$ a constant growth rate [similar to growth

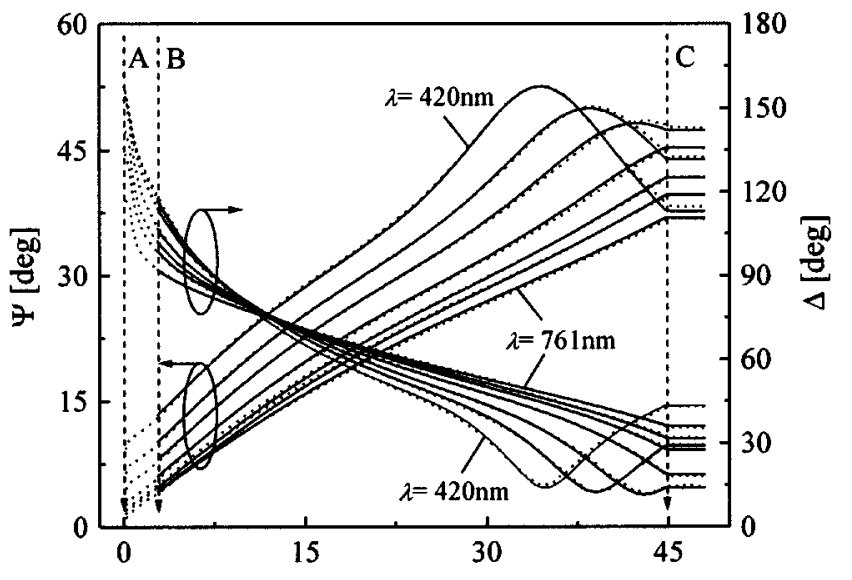

(a)

Time in Minutes

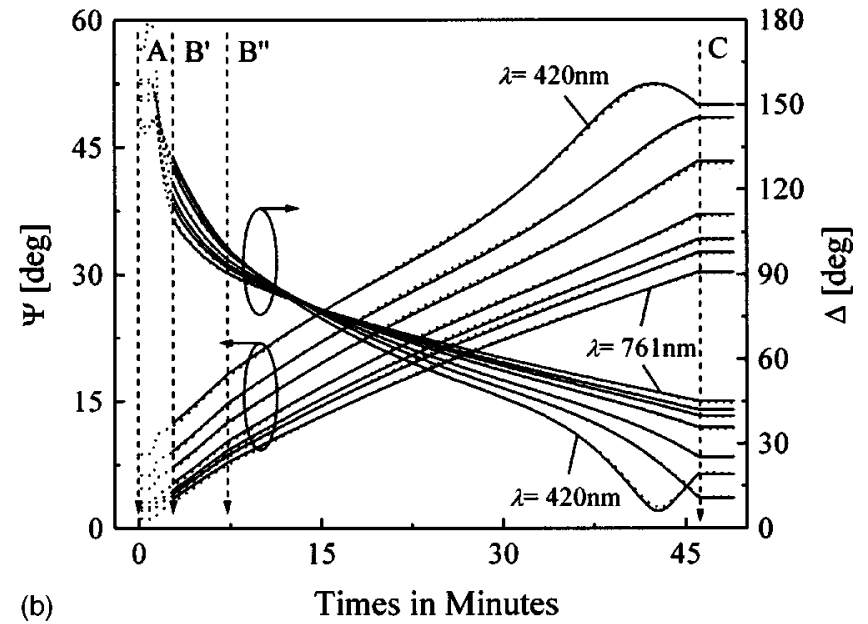

FIG. 2. (a) Experimental (dashed line), and calculated (solid line) in situ spectroscopic ellipsometry $\Psi$ and $\Delta$ spectra of a pure $h$-BN thin-film growth $\left(\mathrm{BN51}: T_{g}=100^{\circ} \mathrm{C}, E_{\text {ion }}=300 \mathrm{~V}, j_{\text {ion }}=55 \mu \mathrm{A} \mathrm{cm}^{-2}, \varphi=74.3^{\circ}\right)$. To avoid confusion, the time dependence of eight wavelengths are shown only. The generated data were calculated using a single-layer growth model. The bestfit parameters are the $h$-BN refractive index $n_{h}$, the layer growth rate $g_{h}$, the final thickness $d_{h}$, and the linear thickness nonuniformity $\delta d / d$. The ion beams were initiated at $t \sim 0(A)$. Between $A$ and $B$, the substrate surface is modified, and formation of a $a-\mathrm{BN}$ nucleation layer occurred. The growth of a homogeneous layer started at $B$. The time slice at $t \sim 2.5 \mathrm{~min}$ was taken for calculation of the virtual interface. Ion sputter and assist beams were turned off at $t \sim 45 \min (C)$. (b) Experimental (dashed line), and calculated (solid line) in situ spectroscopic ellipsometry $\Psi$ and $\Delta$ spectra of a mixed-phase $c / h$-BN thin-film growth $\left(\mathrm{BN} 54: T_{g}=400^{\circ} \mathrm{C}, \quad E_{\text {ion }}=300 \mathrm{~V}, \quad j_{\text {ion }}\right.$ $\left.=55 \mu \mathrm{A} \mathrm{cm}^{-2}, \varphi=74.3^{\circ}\right)$. The generated $\Psi$ and $\Delta$ data were calculated using a two-layer growth model which accounts for the $h$-BN nucleation layer, and the $c$-BN layer growth. The best-fit parameters are refractive indices $\left(n_{h}, n_{c}\right)$, growth rates $\left(g_{h}, g_{c}\right)$, final thicknesses $d$ and linear thickness nonuniformity $\delta d / d$. The growth process was started at $t \sim 0(A)$, and the substrate surface is modified until $B^{\prime}$. The virtual interface was set at $B^{\prime}$. Between $B^{\prime}$ and $B^{\prime \prime}$ the $h$-BN nucleation layer growth was observed which changed into the $c$-BN layer growth at $B^{\prime \prime}$. Ion sputter and assist sources were turned off again after $\sim 45 \mathrm{~min}(C)$, and the film growth stopped. As for the $h$-BN sample, the process parameters were kept constant between $A$ and $C$. In this case, however, two films, a $15 \mathrm{~nm}$ thick pure $h$-BN and a $50 \mathrm{~nm}$ thick mixed-phase $h / c$-BN film were deposited.

initialization at $B$ in Fig. 2(a)] was detected. After about 8 $\min \left(B^{\prime \prime}\right)$ the growth mode changed, and a second layer was deposited at a different growth rate, and with slightly different optical constants. This can be seen in Fig. 2(b) by a slight change of the slope of the $\Psi$ and $\Delta$ spectra. Here, the trans- 
TABLE I. Best-fit results for the BN thin-film model parameters of pure $h$-BN and mixed-phase $h / c$-BN samples. The thickness nonuniformity $\delta d / d$ is the linear deviation from the average thickness of the wedge-shaped thin-film samples over the measurement spot size of $20 \mathrm{~mm}$.

\begin{tabular}{|c|c|c|c|c|c|c|c|c|}
\hline \multirow[b]{2}{*}{ Sample } & \multirow[b]{2}{*}{$\begin{array}{c}T_{g} \\
\left({ }^{\circ} \mathrm{C}\right)\end{array}$} & \multicolumn{3}{|c|}{$h$-BN layer } & \multicolumn{3}{|c|}{$c$-BN layer } & \multirow[b]{2}{*}{$\begin{array}{c}\delta d / d \\
(\%)\end{array}$} \\
\hline & & $\begin{array}{c}d_{h} \\
(\mathrm{~nm})\end{array}$ & $\begin{array}{c}g_{h} \\
\left(10^{-3} \stackrel{\AA}{\AA} \mathrm{s}^{-1}\right)\end{array}$ & $n_{h}$ & $\begin{array}{c}d_{c} \\
(\mathrm{~nm})\end{array}$ & $\begin{array}{c}g_{c} \\
\left(10^{-3} \AA \mathrm{s}^{-1}\right)\end{array}$ & $n_{c}$ & \\
\hline BN51 & 100 & $81 \pm 2$ & $310 \pm 5$ & $1.90 \pm 0.01$ & - & - & - & $13 \pm 1$ \\
\hline BN50 & 200 & $74 \pm 2$ & $280 \pm 5$ & $1.95 \pm 0.01$ & - & - & - & $10 \pm 1$ \\
\hline BN59 & 300 & $75 \pm 3$ & $270 \pm 5$ & $1.90 \pm 0.01$ & - & - & - & $10 \pm 1$ \\
\hline BN54 & 400 & $15 \pm 2$ & $300 \pm 5$ & $1.80 \pm 0.01$ & $50 \pm 2$ & $220 \pm 2$ & $2.00 \pm 0.01$ & $5 \pm 1$ \\
\hline BN53 & 500 & $17 \pm 2$ & $310 \pm 5$ & $1.85 \pm 0.01$ & $46 \pm 1$ & $200 \pm 1$ & $2.00 \pm 0.01$ & $5 \pm 1$ \\
\hline BN55 & 600 & $15 \pm 3$ & $300 \pm 5$ & $1.80 \pm 0.01$ & $45 \pm 2$ & $200 \pm 1$ & $2.00 \pm 0.01$ & $7 \pm 1$ \\
\hline BN56 & 700 & $17 \pm 3$ & $300 \pm 5$ & $1.80 \pm 0.01$ & $42 \pm 3$ & $190 \pm 1$ & $2.10 \pm 0.01$ & $9 \pm 1$ \\
\hline BN57 & 800 & $14 \pm 2$ & $300 \pm 5$ & $1.80 \pm 0.01$ & $45 \pm 2$ & $190 \pm 1$ & $2.05 \pm 0.01$ & $12 \pm 1$ \\
\hline
\end{tabular}

formation from the $h$ - $\mathrm{BN}$ to the $c$ - $\mathrm{BN}$ layer growth occurs. The ion sputter and assist sources were turned off again after $\sim 45 \min (C)$, and film growth stopped. Similar to the $h$-BN sample, the process parameters were kept constant between $A$ and $C$. In this case, however, two films, a $15 \mathrm{~nm}$ thick pure $h$-BN and a $50 \mathrm{~nm}$ thick mixed-phase $h / c$-BN film were deposited.

The best-fit data shown in Fig. 2(a) and 2(b) were calculated using a single-layer model for pure $h$ - BN, and a two-layer model for mixed-phase $h / c$-BN. Because of the deposition geometry (Fig. 1), BN thin films posses nonuniform thicknesses. Film thickness varied linearly over the measurement spot size $(10 \mathrm{~mm}$ diameter $)$ by about $10 \%$. This nonuniformity was considered during model calculations. ${ }^{32}$ Due to the narrow spectral range investigated, the wavelength dependencies of $h$ - and $c$-BN optical constants were neglected. Only the first of the three Cauchy coefficients, i.e., the constant terms were used. ${ }^{20}$ The $h$-BN optical response was recently investigated by ex situ multiple angle-of-incidence spectroscopic ellipsometry, and found to be anisotropic. ${ }^{19,20}$ This anisotropy, however, is difficult to observe at the single angle of incidence used for in situ ellipsometry. The optical responses of both $h$ - BN $\left(n_{h}\right)$, and $c$ - BN $\left(n_{c}\right)$ are treated as isotropic in this work. Besides the final thickness $d$ of a layer at the end of the growth process, two parameters were necessary to model each $\mathrm{BN}$ film, the refractive index $n$ and the growth rate $g$. The latter was assumed to be constant for each layer. As mentioned above, the VI approximation was used to account for the modified interface properties of the substrate. The VI was set at 2.5 min after the beginning of each deposition process. The $\Psi$ and $\Delta$ spectra at that time were converted into pseudodielectric function values, ${ }^{33}$ and varied during the best-fit regression analysis. In this approach, the modification of the silicon/silicon dioxide surface, caused by ion irradiation, as well as the unmodified silicon substrate, form a virtual substrate material. The dielectric function of the virtual substrate contains the optical properties of the silicon substrate at growth temperature, and the dielectric properties of the amorphous layer. The temperature dependence of the $h$-BN and $c$-BN optical constants were also neglected within our model calculation. We determined previously that the BN optical constants remain unchanged between room temperature and $T_{g}=600{ }^{\circ} \mathrm{C} .{ }^{34}$ During the best-fit regression analy- sis the following parameters were varied until the calculated and measured real-time $\Psi$ and $\Delta$ spectra matched as close as possible: thickness $d$, optical constants $n$, and growth rate $g$ of each layer, VI model optical constants, and overall thickness nonuniformity $\delta d / d$. Note that the assumptions of constant refractive indices and growth rates constitute a very strict condition on the model behavior of $\Psi$ and $\Delta$ : any two data points at arbitrary but different times define $g$ and $n$ during growth of the respective layer. Therefore, the perfect agreement between calculated and generated data in Figs. 2(a) and 2(b) within the total growth cycles $[B-C$ in Fig. 2(a) and $B^{\prime}-B^{\prime \prime} ; B^{\prime \prime}-C$ in Fig. 2(b)] indicates a constant growth mechanism for our samples. The resulting best-fit model parameters are summarized in Table I.

The BN thin-film growth parameters obtained by in situ ellipsometry were compared to microstructure investigations performed on polycrystalline BN thin films using HRTEM. In Figs. 3(a) and 3(b) the HRTEM micrographs of samples BN51 and BN54 are shown, respectively. Micrographs were taken from a cross section at the interface between the silicon substrate and BN thin film. Dashed line $A$ indicates the single crystalline silicium substrate interface. An approximately 5 $\mathrm{nm}$ thick amorphous layer, modified by ion mixing was created in the first deposition step. The dashed line $B$ [Fig. 3(a)], $\left[B_{1}\right.$, Fig. 3(b)] symbolizes the top of the $a$-BN layer. Here the virtual interface was set to model the follow-up BN thinfilm growth during ellipsometry data regression. The $h$-BN layer is highly ordered with $c$-axes texture perpendicular to the thin-film normal [Fig. 3(a)]. In Fig. 3(b) the dashed line $\left(B_{2}\right)$ indicates the change from $h$-BN to $c$-BN during growth. The $c$ axes within the $h$-BN nucleation layer in Fig. 3(b) again show a preferential orientation perpendicular to the film normal. We could not observe a $c$-BN grain texture. The thickness of the $h$-BN nucleation layer, obtained by HRTEM, was determined to be $d=13.6 \mathrm{~nm}$, similar to the value obtained by in situ ellipsometry $(d=15 \mathrm{~nm})$.

The phase content of the BN thin films was also determined by IR spectroscopy. Figure 4 shows the IR spectra of samples BN51 and BN54. In the IR spectra of BN51 the two $h$-BN lattice vibrations at $\omega_{\mathrm{TO} h}=775 \mathrm{~cm}^{-1}$ and $\omega_{\mathrm{TO} h}$ $=1395 \mathrm{~cm}^{-1}$ are clearly visible. The IR spectrum from 

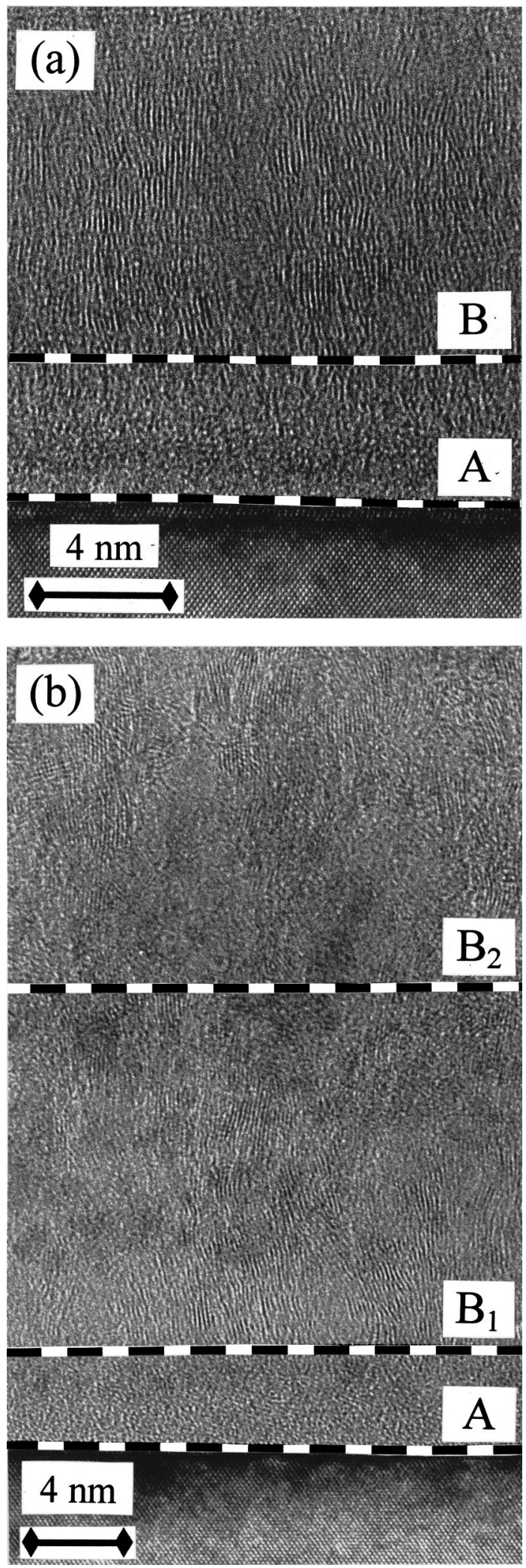

FIG. 3. (a) HRTEM micrograph of sample BN51. The cross section shows the $\mathrm{Si}$ substrate interface $(A)$, an amorphous $\mathrm{BN}$ layer on top of the substrate, and a highly textured $h$-BN layer. No indication of $c$ - BN is observed. The virtual interface which was used for ellipsometry data regression was set at $B$. (b) Same as (a) for sample BN54. The cross section reveals the $\mathrm{Si}$ substrate interface $(A)$, an amorphous BN layer on top of the substrate, a highly textured $h$-BN nucleation layer, and a $c$-BN layer. The dashed line marked with $B_{1}$ indicates the virtual interface during ellipsometry data regression. The transition of $h$-BN to $c$ - BN layer growth occurred at $B_{2}$. The thickness of the $h$-BN nucleation layer observed here is $\sim 13.6 \mathrm{~nm}$.

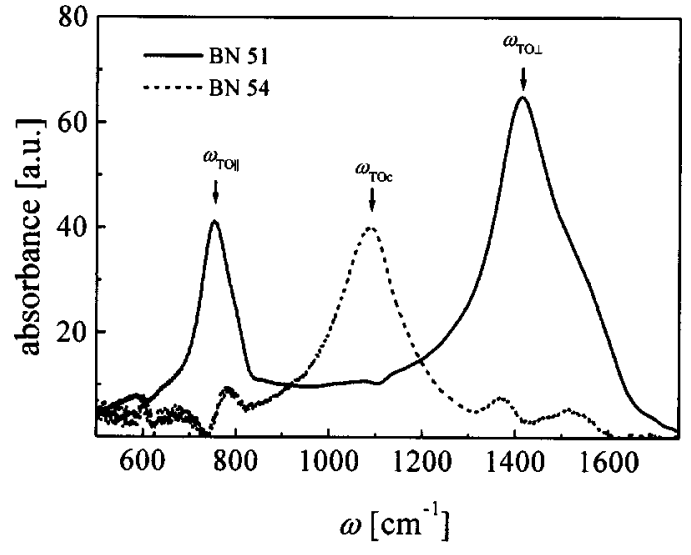

FIG. 4. FTIR transmission spectra taken from samples BN51 and BN54. In agreement with the HRTEM and in situ ellipsometry results, the FTIR data of BN51 indicate the $h$-BN modification only, the spectrum of BN54 reveals the characteristic $h$-BN and $c$-BN lattice absorption bands.

BN54 reveals to the strong $c$-BN absorption at $\omega_{\mathrm{TO}}$ $=1080 \mathrm{~cm}^{-1}$, and two weak $h$-BN absorptions at $\omega_{\mathrm{TO}} h$ $=775 \mathrm{~cm}^{-1}$ and $\omega_{\mathrm{TO} h}=1395 \mathrm{~cm}^{-1}$. As observed by ellipsometry and HRTEM, BN51 mainly contains the $h$-BN modification, whereas BN54 consists of both the $h$-BN and the $c$-BN phase.

Figure 5 shows the $h$ - $\mathrm{BN}$ and $c$-BN layer growth rate as a function of deposition temperature. In the temperature region between 100 and $300^{\circ} \mathrm{C}$ we observed only one-layer $h$-BN film growth, and the $h$-BN growth rate decreases slightly with increasing temperature. Between 400 and $800^{\circ} \mathrm{C}$ we found a two-layer growth. While the growth rate of the $h$-BN nucleation layer is almost constant here, the $c$-BN layer growth rate decreases at higher temperatures. The BN thin-film thickness nonuniformity $\delta d / d$ shows a minimum between $T_{g}=300^{\circ} \mathrm{C}$ and $T_{g}=500{ }^{\circ} \mathrm{C}$, and increases at lower and higher deposition temperatures (Fig. 6).

The final thin-film thickness decreases at higher temperatures (see inset in Fig. 5). This can be explained by the sticking coefficient for boron, which decreases with increasing temperature. ${ }^{35}$ The $h$-BN nucleation layer growth, how-

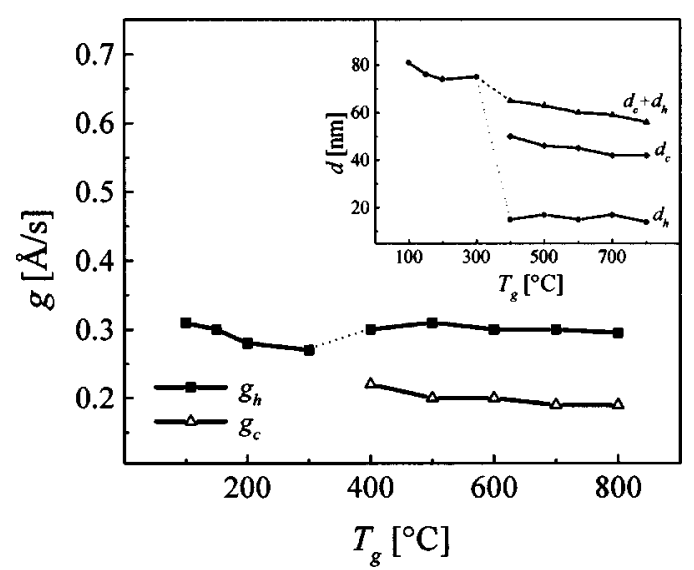

FIG. 5. BN layer growth rates $g$ and film thickness $d$ (inset) vs growth temperature $T_{g}$. The total film thickness decreases with increasing temperatures. Whereas the $h$-BN nucleation layer growth rate is nearly constant, the $c$-BN layer growth rate decreases slightly at higher temperatures. 


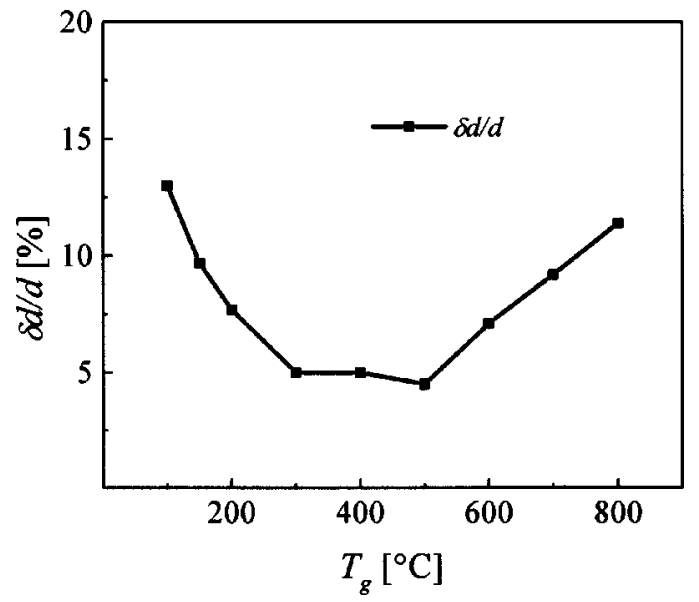

FIG. 6. Thickness nonuniformity $\delta d / d$ as a function of growth temperature. The thin films exhibit best thickness homogeneity at $300^{\circ} \mathrm{C} \leqslant T_{g}$ $\leqslant 500^{\circ} \mathrm{C}$.

ever, is not affected by the deposition temperature. The total film thickness in the region of the two-layer growth is controlled by the $c$-BN layer growth rate.

Recently, Hofsäss et al. ${ }^{36}$ developed a cylindrical thermal spike growth model for ion deposition of diamond-like carbon. This model was extended by the same authors to explain ion-beam assisted $c$-BN thin-film growth. This model is based on a combination of thermal spikes and ion subplantation processes. The bombarding ions penetrate beneath the growing film surface. After a series of elastic collisions with as-deposited atoms, the residual energy of the penetrating ions is converted into lattice phonons within a small volume (spike volume). The temperature within this spike volume can reach more than $1000 \mathrm{~K}$, which may be sufficient to activate a recrystallization process. Accordingly, the $c$-BN growth occurs via rearrangement of the originally deposited $s p^{2}$-bonded boron and nitrogen atoms into tetrahedral coordination within the spike volume. Within the cylindrical spike model the transformation from $h$-BN to $c$ - BN layer growth, and therefore the $h$-BN nucleation layer thickness, is not a function of substrate temperature because of much higher temperatures within the thermal spike volume. Furthermore this model can also explain the $s p^{2}$-bonded BN material between the $c$ - $\mathrm{BN}$ grains, and small grain sizes. However the cylindrical thermal spike model does not account for the $c$-BN nucleation process.

The formation of the $h$-BN nucleation layer in advance of the $c$-BN growth was suggested by Hofsäss et al. ${ }^{36}$ to occur in order to provide a suitable surface for the $c$-BN nucleation. A highly oriented hexagonal nucleation layer seems to be essential for the subsequent $c$-BN growth, because textured $h$-BN with $c$ axis perpendicular to the thinfilm normal allows 2:3 epitaxial lattice match between (0002) $h$-BN and (111) $c$-BN lattice planes. ${ }^{37}$

Very recently, it was reported by Feldermann et al. ${ }^{38}$ that a growth temperature higher than a threshold of $125^{\circ} \mathrm{C}$, combined with a sufficient ion bombardment, is necessary in order to nucleate $c$ - BN. Once $c$ - $\mathrm{BN}$ is nucleated it continues to grow even at room temperature. To serve as a nucleation surface for $c$-BN growth, the $h$-BN layer should have a rea- sonable degree of crystallinity. Elevated growth temperatures can improve crystallinity of the nucleation layer, which explains the general temperature threshold value for $c$-BN nucleation at $125^{\circ} \mathrm{C} .{ }^{36}$

Here we observe in agreement with the cylindrical thermal spike model that the $h$-BN nucleation layer thickness, and therefore the transition from $h$-BN to $c$-BN layer growth, is not a function of growth temperature in the range of two-layer growth from 400 to $800{ }^{\circ} \mathrm{C}$. The lack of $c$-BN nucleation at temperatures below $400{ }^{\circ} \mathrm{C}$ might be caused by too many crystalline imperfections in the $h$-BN layer.

If we consider $c$ - $\mathrm{BN}$ growth due to intrinsic compressive stress we believe that at least the $h$-BN nucleation layer thickness should show a temperature dependence. This could be explained by treating the $h$-BN nucleation layer thickness as that of a critical film thickness. The hydrostatic film stress determines the critical thickness, and scales with temperature via the Poisson ratio. Additionally, if we consider the origin of compressive film stress by a dynamic concentration of interstitials and vacancies, the competition between point defect production and loss should also be a function of temperature. From this point of view we would favor the $c$-BN film growth by thermal spikes rather than by intrinsic compressive stress.

However, our BN films were deposited in a nonoptimized growth regime. Preliminary results from new experiments, which deal with in situ ellipsometry investigations of the nucleation layer thickness as a function of ion energy and ion beam current density, show that thickness of the $h$-BN nucleation layer can be reduced by decreasing growth rates due to ion irradiation. This may also improve crystallinity of the $h$-BN nucleation layer, which would lead to a two-layer $h / c$-BN thin film growth at lower substrate temperatures than shown in this work.

\section{CONCLUSIONS}

In situ spectroscopic ellipsometry is a powerful technique for real-time study of BN thin-film layer growth. We have shown that the BN thin-film microstructure observed by in situ ellipsometry investigations is confirmed by electron microscopy. We are able to monitor the growth process, including the phase transition from $h$ - $\mathrm{BN}$ to $c$ - $\mathrm{BN}$ growth, using real-time ellipsometric spectra. BN model-growth parameters obtained from data regression of the in situ ellipsometric data are consistent with results from HRTEM and IR measurements.

The $c$-BN thin-film nucleation and growth process is discussed within the cylindrical thermal spike model. We found that this model can explain the $c$-BN growth. Our results support the assumption that the formation of the $h$ - BN nucleation layer is necessary to provide a surface for epitaxial $c$-BN growth. It may be possible to deposit $c$-BN thin films avoiding the $h$-BN intermediate layer by using a suitable substrate material. 


\section{ACKNOWLEDGMENTS}

The authors wish to acknowledge T. Scherzer and U. Decker, Institute of Surface Modification, for technical support during IR measurements.

${ }^{1}$ P. B. Mirkarimi, K. F. McCarty, and D. L. Medlin, Mater. Sci. Eng., R. R21, 47 (1997).

${ }^{2}$ T. Ichiki, T. Momose, and T. Yoshida, J. Appl. Phys. 75, 1330 (1994).

${ }^{3}$ M. Kuhr, S. Reinke, and W. Kulisch, Surf. Coat. Technol. 74-75, 806 (1995).

${ }^{4}$ K. S. Park, D. Y. Lee, K. J. Kim, and D. W. Moon, Appl. Phys. Lett. 70, 315 (1997).

${ }^{5}$ J. Hahn, M. Friedrich, R. Pintaske, M. Schaller, N. Kahl, D. R. T. Zahn, and F. Richter, Diamond Relat. Mater. 5, 1103 (1996).

${ }^{6}$ H. Hofsäss, C. Ronning, U. Griesmeier, M. Gross, S. Reinke, and M. Kuhr, Appl. Phys. Lett. 67, 46 (1995).

${ }^{7}$ T. A. Friedmann et al. J. Appl. Phys. 76, 3088 (1994).

${ }^{8}$ D. J. Kester, K. S. Ailey, D. J. Lichtenwalner, and R. F. Davis, J. Vac. Sci. Technol. A 12, 3074 (1994).

${ }^{9}$ W. L. Zhou, Y. Ikuhara, M. Murakawa, S. Watanabe, and T. Suzuki, Appl. Phys. Lett. 66, 2490 (1995).

${ }^{10}$ G. Sené, D. Bouchier, S. Ilias, M. A. Djouadi, J. Pascallon, V. Stambouli, P. Möller, and G. Hug, Diamond Relat. Mater. 5, 530 (1996).

${ }^{11}$ P. B. Mirkarimi, K. R. McCarty, D. L. Medlin, W. G. Wolfer, T. A. Friedmann, E. J. Klaus, G. F. Cardinale, and D. G. Howitt, J. Mater. Res. 9, 2925 (1994).

${ }^{12}$ C. A. Davis, Thin Solid Films 226, 30 (1992).

${ }^{13}$ J. Robertson, Diamond Relat. Mater. 5, 519 (1996)

${ }^{14}$ D. R. McKenzie, D. J. H. Cockayne, D. A. Muller, M. Murakawa, S. Miyake, S. Watanabe, and I. S. Falconer, J. Appl. Phys. 70, 3007 (1991).

${ }^{15}$ S. Reinke, M. Kuhr, W. Kulisch, and R. Kassing, Diamond Relat. Mater. 4, 272 (1994).

${ }^{16}$ C. Weißmantel, K. Bewilogua, D. Dietrich, H.-J. Erler, H.-J. Hinneberg, S. Klose, W. Nowick, and G. Reisse, Thin Solid Films 72, 19 (1980).

${ }^{17}$ H. Hofsäss, M. Feldermann, M. Sebastian, and C. Ronning, Phys. Rev. B 55, 13230 (1997).
${ }^{18}$ R. M. A. Azzam and N. M. Bashara, Ellipsometry and Polarized Light (North-Holland, Amsterdam, 1984).

${ }^{19}$ M. Schubert, B. Rheinländer, E. Franke, H. Neumann, J. Hahn, M. Röder, and F. Richter, Appl. Phys. Lett. 70, 1819 (1997).

${ }^{20}$ E. Franke, M. Schubert, H. Neumann, T. E. Tiwald, D. W. Thompson, J. A. Woollam, J. Hahn, and F. Richter, J. Appl. Phys. 82, 2906 (1997).

${ }^{21}$ O. Stenzel, J. Hahn, M. Röder, A. Ehrlich, S. Prause, and F. Richter, Phys. Status Solidi A 158, 281 (1996).

${ }^{22}$ R. Poerschke and O. Madelung, Semiconductor Group IV and III-V Compounds (Springer, Berlin, 1991).

${ }^{23}$ R. W. Collins, I. An, H. V. Nguyen, and Y. Lu, Thin Solid Films 233, 244 (1994).

${ }^{24}$ R. Geick, C. H. Perry, and G. Rupprecht, Phys. Rev. 146, 543 (1966).

${ }^{25}$ P. J. Gielisse, S. S. Mitra, J. N. Plendl, R. D. Griffis, L. C. Mansur, R. Marshall, and E. A. Pascoe, Phys. Rev. 155, 1039 (1967).

${ }^{26}$ E. Franke, H. Neumann, M. Zeuner, W. Frank, and F. Bigl, Surf. Coat. Technol. 97, 90 (1997)

${ }^{27}$ E. Franke, PhD thesis, University Leipzig, 1998.

${ }^{28}$ G. E. Jellison, Thin Solid Films 313-314, 33 (1998), and references therein.

${ }^{29}$ E. D. Palik, Handbook of Optical Constants of Solids (Academic, New York, 1998).

${ }^{30}$ H. Yao, P. G. Snyder, and J. A. Woollam, J. Appl. Phys. 70, 3261 (1991).

${ }^{31}$ D. E. Aspnes, J. Vac. Sci. Technol. A 14, 960 (1996).

${ }^{32}$ Guide to Using WVASE32 (Woollam Co., Lincoln, NE, 1995).

${ }^{33}$ D. E. Aspnes and A. A. Studna, Phys. Rev. B 27, 985 (1983).

${ }^{34}$ E. Franke, M. Schubert, J.-D. Hecht, H. Neumann, T. E. Tiwald, D. W. Thompson, H. Yao, J. A. Woollam, and J. Hahn, J. Appl. Phys. 84, 526 (1998).

${ }^{35}$ K.-L. Barth, A. Lunk, and J. Ulmer, Surf. Coat. Technol. 92, 96 (1997).

${ }^{36}$ H. Hofsäss, H. Feldermann, R. Merk, M. Sebastian, and C. Ronning, Appl. Phys. A: Solids Surf. 66, 153 (1998).

${ }^{37}$ J. Widany, Th. Frauenheim, and W. L. Lambrecht, J. Mater. Chem. 6, 899 (1996).

${ }^{38}$ H. Feldermann, R. Merk, H. Hofsäss, C. Ronning, and T. Zheleva, Appl. Phys. Lett. 74, 1552 (1999). 